\title{
Non-Compact Symmetric Spaces and the Toda Molecule Equations
}

\author{
L. A. Ferreira ${ }^{\star}$ and D. I. Olive \\ The Blackett Laboratory, Imperial College, London SW7 2BZ, England
}

\begin{abstract}
It has been shown by Olshanetsky and Perelomov that the Toda molecule equations associated with any Lie group $G$ describe special geodesic motions on the Riemannian non-compact symmetric space which is the quotient of the normal real form of $G, G^{N}$, by its maximal compact subgroup. This is explained in more detail and it is shown that the "fundamental Poisson bracket relation" involving the Lax operator $A$ and leading to the Yang-Baxter equation and integrability properties is a direct consequence of the fact that the Iwasawa decomposition for $G^{N}$ endows the symmetric space with a hidden group theoretic structure.
\end{abstract}

\section{Introduction}

Integrable systems are currently of great interest for a variety of reasons, mathematical and physical $[1,2]$. One reason is that some four dimensional gauge theories, perhaps spontaneously broken by a Higgs mechanism may belong to a new class of integrable theory. The integrability may relate to the electromagnetic duality conjectures [3] whose validity is most favoured in the $N=4$ supersymmetric gauge theories [4]. It is known that the radial dependence of certain spherically symmetric monopole solutions occurring in such theories is governed by a Toda molecule equation $[5,6]$ (with $t$ replaced by $i$ times radius):

$$
\frac{d^{2}}{d t^{2}} \phi_{a}=-\exp \left(\sum_{b} K_{a b} \phi_{b}\right), \quad a=1,2, . . r .
$$

These equations are known to be completely integrable when the square, non-singular, matrix $K$ is the Cartan matrix for a simple Lie group $G$ (of rank $r$ ). The solutions to these equations are also known $[7,8,9,6]$.

\footnotetext{
* Supported by $\mathrm{CNP}_{q}$ (Brasil)
} 
Olshanetsky and Perelomov [8] constructed these solutions from an interesting geometrical picture in which they viewed Eq. (1.1) as describing a projection of certain geodesic motions on a symmetric space $G^{N} / K$ explained below. It is our purpose to understand the details of how this is done more clearly and to see how the integrability properties arise in this picture. The reason this is important to do is that Olshanetsky and Perelomov have conjectured that a similar picture of geodesic motions on symmetric spaces (suitably generalized) may underly all integrable systems $[8,10]$. This is almost certainly true for a large class and Eqs. (1.1) constitute a relatively simple prototype class of theory. Related but more complicated theories would involve two rather than one dimension and KacMoody or higher algebras rather than finite dimensional Lie algebras.

The results we shall find depend on some very special properties of the type of symmetric space considered as we now explain. The complex Lie group whose Cartan matrix appears in (1.1) has a non-compact real form $G^{N}$ ("the normal" form) whose generators are the Cartan subalgebra generators $H_{i}$ and the step operators $E_{\alpha} \cdot G^{N}$ has a maximal compact subgroup $K$, whose generators are $E_{\alpha}-E_{-\alpha}$ (for all roots $\alpha$ ). The relevant symmetric space is $G^{N} / K$ and is automatically non-compact and Riemannian. It has a very special structure owing to the fact that its points can be parametrised using the Iwasawa decomposition [11] which states that any point of $G^{N}$ can be uniquely expressed as a product of three factors

$$
g=n a k,
$$

where $n$ is obtained by exponentiating a real linear combination of the $E_{\alpha}$ for positive roots $\alpha, a$ by exponentiating a real linear combination of the $H_{i}$ and $k$ is in $K$. It follows that the quantities $n a$ can be used to parametrise the cosets of $G^{N}$ which constitute the points of the symmetric space $G^{N} / K$. The possible quantities $n a$ form a group B (the Borel subgroup of $G^{N}$ ) whose Lie algebra b will be relevant for the integrability. By scaling the real parameters in the exponents to zero we see that the space $G^{N} / K$ can be continuously contracted to a point. Thus the symmetric space $G^{N} / K$ has its own group theoretical structure, B, and is topologically trivial. It thus differs in an essential way from the types of symmetric space considered by some other authors [12]. Nevertheless it is interesting that such spaces arise naturally in supergravity theories. Thus $E_{7,7} / \mathrm{SU}(8)$ is an example [13].

Given a symmetric space $G^{N} / K$ there exists an automorphism $\sigma$ of $G^{N}$ such that the generators of $K$ are even under $\sigma$ while the remaining (orthogonal) generators are odd $\left(\operatorname{so} \sigma^{2}=1\right)$. Here

$$
\sigma\left(H_{i}\right)=-H_{i}, \quad \sigma\left(E_{\alpha}\right)=-E_{-\alpha} .
$$

Let us define

$$
g^{-1} \frac{d g}{d t}=A+i B
$$

where $A$ and $B$ are odd and even respectively under $\sigma$. Then in Sect. 2 we show 
that the Lagrangian for the geodesic motion is

$$
\mathscr{L}=\operatorname{Tr}\left(A^{2}\right) / 2
$$

and that the corresponding equation of motion

$$
\frac{d A}{d t}+i[B, A]=0
$$

takes the form of a Lax pair equation.

The Lagrangian (1.5), and, more importantly, the corresponding Hamiltonian, are automatically positive. This is a reflection of the Riemannian nature of the symmetric space. If we chose $k=1$ in (1.2) which means that we are parametring the point of the symmetric space by an element of $\mathbf{B}$, then (1.4) can be expanded in terms of generators of $\mathbf{B}$ with coefficients which are functions of the coordinates and momenta.

In Sect. 3 we explain how to evaluate the Poisson bracket algebra of these coefficients by using a refined version of Noether's Theorem. Since these coefficients turn out to be Noether charges for right transformations

$$
g \rightarrow g^{\prime}=g b, \quad g, b \in \mathbf{B},
$$

their Poisson bracket algebra is the Lie algebra of $\mathbf{B}$ (even though these quantities are only partially conserved by virtue of (1.6)).

In particular we find in Sect. 4 what Faddeev calls the Fundamental Poisson Relation (FPR) [1]

$$
\{A+i B \otimes A+i B\}_{P B}=-[\mathbb{P},(A+i B) \otimes 1+1 \otimes(A+i B)],
$$

where $\mathbb{P}$ is the quantity previously constructed by Turok and one of us [14]. We also explain that because of a similarity in structure between $\mathbb{P}$ and $A+i B$, Eq. (1.8) can be written in the form of a classical Yang-Baxter equation $[15,16]$.

The next stage in Sect. 5 is to show how the geodesic motion can be constrained in a consistent way to yield the Toda equation (1.1), with the Lax and FPR equations (1.6) and (1.8) reducing to their known forms appropriate to (1.1). This completes our demonstration of how the geometrical picture of Olshanetsky and Perelomov leads to the structures common to integrable systems. Possibilities for generalisation are discussed in the conclusion.

\section{Geodesic Motion}

Much of our work applies to the geodesic motion of a particle moving on any Riemanian non-compact symmetric space, and we shall therefore postpone specialization to the special type described in the introduction to Sect. 4. It is this type which leads to the Toda equations (1.1) when appropriate constraints are made. Let $F$ denote any non-compact simple Lie group furnished with a Cartan involution $\sigma$ [11]. That $\sigma$ is an involution means that it is an automorphism of $F$ satisfying

$$
\sigma^{2}=1
$$


The generators of the Lie algebra $\mathbf{f}$ of $F$ can be split into two subspaces even or odd under $\sigma$ (by 2.1):

$$
\mathbf{f}=\mathbf{k}+\mathbf{p}, \quad \sigma(\mathbf{p})=-\mathbf{p}, \quad \sigma(\mathbf{k})=\mathbf{k} .
$$

It follows that as $\sigma$ is also an automorphism of $\mathbf{f}$,

$$
[\mathbf{k}, \mathbf{k}] \subset \mathbf{k}, \quad[\mathbf{k}, \mathbf{p}] \subset \mathbf{p}, \quad[\mathbf{p}, \mathbf{p}] \subset \mathbf{k} .
$$

If $T$ and $S$ are elements of $\mathbf{f}$ we have a $\sigma$ invariant Killing form which we write as $\operatorname{Tr}(T S)=\operatorname{Tr}(\sigma(T) \sigma(S))$. The Cartan property is that (in our notation)

So, by (2.2),

$$
\operatorname{Tr}(T \sigma(S)) \text { is negative definite. }
$$

$$
\operatorname{Tr}(T S)\left\{\begin{array}{l}
\text { is positive definite if } T, S \in \mathbf{p} \\
\text { is negative definite if } T, S \in \mathbf{k} \\
\text { is zero if } T \in \mathbf{p} \text { and } S \in \mathbf{k} .
\end{array}\right.
$$

k generates a subgroup $K \subset F$ which is a maximal compact subgroup. We think of the remaining generators $\mathbf{p}$ as "non-compact" generators. $K$ is also the subgroup of $F$ invariant with respect to $\sigma$. For any element $g \in F$ we define a "principal variable" $[8,17,18]$ :

$$
x(g)=g \sigma(g)^{-1} \text { so } \sigma(x)=x^{-1}
$$

As $x(g k)=x(g)$ if $k \in K$, we see that $x$ is actually defined on the cosets $F / K$ which constitute the points of the symmetric space $F / K$. In fact, there is a unique correspondence between the cosets and the point $x$, and we are going to regard $x$ as one way of labelling a point of the symmetric space. The geodesic equation of motion for a particle on the symmetric space is

$$
\frac{d}{d t}\left(x^{-1} \dot{x}\right)=0 \text {. }
$$

We shall show that the Lagrangian leading to this can be written

$$
\mathscr{L}=\operatorname{Tr}\left(x^{-1} \dot{x}\right)^{2} / 8 .
$$

In order to do this it is useful to define

$$
g^{-1} \dot{g}=A+i B, \quad A \in \mathbf{p}, \quad B \in \mathbf{k},
$$

so that

$$
A=\left(g^{-1} \dot{g}-\sigma\left(g^{-1} \dot{g}\right)\right) / 2, \quad i B=\left(g^{-1} \dot{g}+\sigma\left(g^{-1} \dot{g}\right)\right) / 2 .
$$

Then it is easy to check that

$$
x^{-1} \dot{x}=2 \sigma(g) A \sigma(g)^{-1} .
$$

Hence by (2.8) we can write the Lagrangian in the alternative form

$$
\mathscr{L}=\operatorname{Tr}\left(A^{2}\right) / 2 .
$$

According to (2.5) and (2.9) the Lagrangian is therefore positive, a reflection of the 
Riemannian nature of the space. The Hamiltonian will likewise be positive, thus assuring a desirable physical feature. Differentiating (2.11)

$$
\frac{d}{d t}\left(x^{-1} \dot{x}\right)=2 \sigma(g)(\dot{A}+i[B, A]) \sigma(g)^{-1} .
$$

This shows that the geodesic equation of motion (2.7) is equivalent to a "Lax pair" equation [19]

$$
\dot{A}+i[B, A]=0 .
$$

As $A \in \mathbf{p}$ (Eq. 2.9) this equation has precisely as many components as the particle has degrees of freedom, namely $\operatorname{dim} \mathbf{p}=\operatorname{dim}(F / K)$, and is therefore the natural way of writing the equation of motion in preference to (2.7), the components of which contain redundancies as they number $\operatorname{dim} \mathbf{f}$.

So far we have not assigned a unique element $g$ to each coset and if we change our choice: $g \rightarrow g^{\prime}=g k$, we find that $A$ and $B$ change accordingly;

$$
A \rightarrow A^{\prime}=k^{-1} A k, \quad B \rightarrow B^{\prime}=k^{-1} B k+k^{-1} \dot{k} .
$$

Thus $B$ transforms as a $K$ gauge potential and the Lax equation (2.14) states that $A$ is covariantly constant. Notice that the Lagrangian (2.8) and (2.12) is $K$ gauge invariant.

Let us vary $g$ infinitesimally by a right translation

$$
\delta g=g R,
$$

where for the time being $R \in \mathbf{f}$ and is small. Then

$$
\delta\left(g^{-1} \dot{g}\right)=\dot{R}-\left[R, g^{-1} \dot{g}\right],
$$

and the response of the Lagrangian (2.12) is

$$
\delta \mathscr{L}=\operatorname{Tr}\left(A \delta\left(g^{-1} \dot{g}\right)\right)=\operatorname{Tr}(\dot{R} A-i R[B, A])=\frac{d}{d t} \operatorname{Tr}(R A)-\operatorname{Tr}(R(\dot{A}+i[B, A])) .
$$

If we consider $R$ as an Euler Lagrange variation we conclude that $\mathscr{L}$ is indeed the correct Lagrangian as it yields the correct equation of motion (2.14).

Now vary $g$ infinitesimally by a left variation

$$
\delta g=L g,
$$

where for the time being $L \in \mathbf{f}$ and is small. We find from (2.6) that

$$
\begin{gathered}
\delta x=L x-x \sigma(L), \\
\delta\left(x^{-1} \dot{x}\right)=\left[\sigma(L), x^{-1} \dot{x}\right]+x^{-1} \dot{L} x-\sigma(\dot{L}) .
\end{gathered}
$$

As

$$
\sigma\left(x^{-1} \dot{x}\right)=-\dot{x} x^{-1},
$$

we find that the response of the Lagrangian in the form (2.8) is

$$
\delta \mathscr{L}=\operatorname{Tr}\left(\dot{x} x^{-1} \dot{L}\right) / 2=-\operatorname{Tr}\left(x^{-1} \dot{x} \sigma(\dot{L})\right) / 2
$$


Thus if $L$ is an Euler-Lagrange variation we find the Euler-Lagrange equation of motion in the form (2.7).

We remark that many of the equations derived so far, for example the Lax equation (2.14), hold for any coset space $F / K$ without assuming the symmetric space or other properties. We now come to the crucial point where the special nature of the symmetric space described matters.

It is explained in Helgason [11] how, given a non-compact simple group $F$ equipped with a Cartan involution $\sigma$, it is possible to construct a maximal abelian subalgebra $\mathbf{a}$ of $\mathbf{p}$ and a maximal nilpotent subalgebra $\mathbf{n}$ such that, according to a result of Iwasawa, there exists a unique decomposition of any element of $F$ into three factors,

$$
g=n a k,
$$

where $n$ and $a$ are obtained by exponentiating $\mathbf{n}$ and a respectively with real coefficients and $k \in K$. If $\sigma$ is the involution (1.3) mentioned in the introduction $\mathbf{a}$ and $\mathbf{n}$ are as specified in (4.3).

Thus given the Iwasawa decomposition (2.24) there exists a "natural" choice of representative from each coset corresponding to each point of the symmetric space, simply $k=1$. There is no analogue of this for the compact symmetric spaces more commonly studied in physics.

In this "gauge" $k=1, g=n a$. Since $\mathbf{n}+\mathbf{a}$ forms a subalgebra of $\mathbf{f}$ this means that the representative points $g$ all belong to the group $\mathbf{B} \subset F$ obtained by exponentiating $\mathbf{b}=\mathbf{n}+\mathbf{a}$. Thus the symmetric space $F / K$ is endowed with a hidden group theoretical structure whose influence on the dynamics of our particles is studied in the next sections.

Given this gauge our Euler Lagrange variations (2.16) should respect it. Thus in (2.16) we should restrict $R \in \mathbf{b}$. As $\operatorname{dim} \mathbf{b}=\operatorname{dim} \mathbf{p}$ this still yields the same Euler Lagrange equation (2.14).

\section{Exact and Broken Symmetries of the Lagrangian}

We now show that the Lagrangian (2.8) or (2.12) exhibits at least three kinds of symmetry, and evaluate the corresponding Noether charges. For certain of these symmetries the Poisson bracket algebra of the associated Noether charges is isomorphic to that of the original infinitesimal variations, despite the fact that in one case the symmetries are not exact but broken. Later we show how these resultant algebras lead to the integrability properties and in particular the Yang-Baxter equations, both for the geodesic motion problem and the Toda molecule equations (1.1).

The analysis depends on a refined version of Noether's Theorem stated in detail and proven in Appendix I. We consider the response of a Lagrangian $\mathscr{L}(q, \dot{q})$ to infinitesimal variations of the coordinates $q_{i}(i=1 \ldots N)$.

$$
\delta q_{i}=\varepsilon(t) F_{i}(q, \dot{q}), \quad \text { and } \quad \delta \mathscr{L}=\frac{d}{d t}(\varepsilon X)+\dot{\varepsilon} Q+\varepsilon D
$$

(with $X, Q$ and $D$ depending on $q$ and $\dot{q}$ only and not $\ddot{q}$ ). 
$Q$, the Noether charge, is read off as the coefficient of $\dot{\varepsilon}$ in (3.1) and it satisfies the partial conservation equation, $\dot{Q}=D$.

We distinguish two interesting special cases which we denote cases $\alpha$ and $\beta$.

Case $\alpha$ is the circumstance that $D$ vanishes in (3.1) so that $Q$ is conserved. $Q$ can then be regarded as the canonical generator generating the original transformation with constant $\varepsilon$ (as this is now canonical).

The second circumstance, case $\beta$, is that the variations $F$ are velocity independent. Then it can be shown that $X$ can be taken to vanish. When this is done for three variations $\delta, \delta^{\prime}$ and $\delta^{\prime \prime}$ of this type which satisfy

$$
\left[\delta, \delta^{\prime}\right] q_{i}=\delta^{\prime \prime} q_{i} \quad\left(\varepsilon^{\prime \prime}=\varepsilon \varepsilon^{\prime}\right)
$$

then

$$
\left\{Q, Q^{\prime}\right\}_{P B}=-Q^{\prime \prime}
$$

Thus, the Poisson bracket algebra of the Noether charges coincides (up to a sign) with the algebra of the original infinitesimal variations, even though the charges may be only partially conserved.

A more complete statement of these results and their proofs appears in Appendix I.

The three types of variation we shall consider in turn are respectively global right translations of $g$ (case $\beta$ ), global left translations of $g$ (case $\alpha$ and $\beta$ ) and time translations (case $\alpha$ ).

The most important symmetry in what follows is the most unexpected one, that due to right translations of $g$. The reason any such symmetry is unexpected is that in general right action on $g$ does not have an unambiguous action on the cosets which are the points of this symmetric space. That is if $g \rightarrow g r, r \in F, g r$ and $g k r$ do not usually lie in the same coset $F / K$. However for this special sort of symmetric space we are considering we can use the Iwasawa decomposition (2.24) as explained in the preceding section to choose a "gauge" $k=1$. This leaves $g$ an element of the group B whose points are thus in precise correspondence with the points of the symmetric space. Right action on $g$ by another element of $\mathbf{B}$ leaves $g$ in $\mathbf{B}$ as $\mathbf{B}$ is a group, thus transporting one point of this symmetric space, $g$, to another, $g b$.

We shall now consider an infinitesimal global version of this,

$$
\delta_{R_{0}} g=\varepsilon(t) g R_{0}, \quad R_{0} \in \mathbf{b}, \quad \dot{R}_{0}=0 .
$$

By Eq. (2.18) the response of the Lagrangian is, as $R=\varepsilon R_{0}, \delta \mathscr{L}=\dot{\varepsilon} \operatorname{Tr}\left(R_{0} A\right)-$ $i \varepsilon \operatorname{Tr}\left(R_{0}[B, A]\right)$ as the variation (3.4) is velocity independent, the conditions of case $\beta$ are satisfied. Comparing with (3.1) we see that already $X=0$ so that we can read off the Noether charge as the coefficient of $\dot{\varepsilon}$ (see Eq. (3.1)):

$$
Q\left(R_{0}\right)=\operatorname{Tr}\left(R_{0} A\right)
$$

As $\left[\delta_{R_{0}}, \delta_{R_{0}^{\prime}}^{\prime}\right] g=g\left[R_{0}, R_{0}^{\prime}\right]=\delta_{\left[R_{0}, R_{0}^{\prime}\right] g}$ when $\varepsilon=1$, we have from (3.2), (3.3) and (3.5)

$$
\left\{\operatorname{Tr}\left(A R_{0}\right), \operatorname{Tr}\left(A R_{0}^{\prime}\right)\right\}_{P B}=-\operatorname{Tr}\left(A\left[R_{0}, R_{0}^{\prime}\right]\right)
$$


This specifies the Poisson brackets of the components of $A$, and is essentially the so-called "fundamental Poisson relation" which will lead to the classical YangBaxter equations as explained in the next section. We emphasize that Eq. (3.6) holds even though these quantities are not conserved. The situation resembles that in particle physics where current algebra relations can be derived even though the currents are not conserved.

Now consider infinitesimal global left translations of $g$, initially preserving the gauge $k=1$ so that

$$
\delta_{L_{0}} g=\varepsilon(t) L_{0} g, \quad L_{0} \in \mathbf{b}, \quad \dot{L}_{0}=0 .
$$

By Eq. (2.23) the response of the Lagrangian is

$$
\delta \mathscr{L}=(1 / 2) \dot{\varepsilon} \operatorname{Tr}\left(\dot{x} x^{-1} L_{0}\right)=-(1 / 2) \dot{\varepsilon} \operatorname{Tr}\left(x^{-1} \dot{x} \sigma\left(L_{0}\right)\right) .
$$

Thus if $\dot{\varepsilon}$ vanishes, the Lagrangian is invariant. Conditions $(\alpha)$ and $(\beta)$ of our Noether's theorem are both satisfied. Comparing with Eq. (3.1) we see that $X$ and $D$ both vanish so that we can read off from (3.8) the coefficient of $\dot{\varepsilon}$ as the conserved charge

$$
X\left(L_{0}\right)=\operatorname{Tr}\left(\dot{x} x^{-1} L_{0}\right) / 2=-\operatorname{Tr}\left(x^{-1} \dot{x} \sigma\left(L_{0}\right)\right) / 2 .
$$

Since when $\varepsilon=1$,

$$
\left[\delta_{L_{0}}, \delta_{L_{0}^{\prime}}\right] g=-\left[L_{0}, L_{0}^{\prime}\right] g=-\delta_{\left[L_{0}, L_{0}^{\prime}\right]} g,
$$

we derive from (3.2) and (3.3) that

$$
\left\{X\left(L_{0}\right), \quad X\left(L_{0}^{\prime}\right)\right\}_{P B}=X\left(\left[L_{0}, L_{0}^{\prime}\right]\right),
$$

where in the first instance $L_{0}$ and $L_{0}^{\prime}$ are both elements of b. Obviously $\left[\delta_{L_{0}}, \delta_{R_{0}}\right] g=0$, when $L_{0}$ and $R_{0}$ are both elements of $\mathbf{b}$. Hence by (3.2) and (3.3),

$$
\left\{X\left(L_{0}\right), \quad \operatorname{Tr}\left(A R_{0}\right)\right\}_{P B}=0 .
$$

Now let us consider the effect of enlarging the class of left and right transformations from $\mathbf{B}$ to $F$ by including elements of $K$. As we commented before, $x$ and the Lagrangian are invariant under right translations $g \rightarrow g k$ even if $k$ depends on time, as long as it is an element of $K$. Hence there is no Noether charge associated with infinitesimal global variations of this kind, and indeed the expression (3.5) vanishes by virtue of (2.5) and (2.9).

On the other hand if we consider global left transformations $g \rightarrow g^{\prime}=l g=n^{\prime} a^{\prime} k^{\prime}$ and then "gauge" $k^{\prime}$ to unity we see from (3.8) that we have a Noether symmetry whenever $\dot{\varepsilon}=0$, and $L_{0}$ is any generator of $\mathbf{f}$, not just b. Thus in Eq. (3.10) the range of allowed $L_{0}$ and $L_{0}^{\prime}$ can be extended from $\mathbf{b}$ to $\mathbf{f}$. Nevertheless left and right transformations of $g$ only commute when both are constrained to B. Hence in Eq. (3.11) the range of $L_{0}$ cannot be extended from b to $\mathbf{f}$.

Finally let us consider time translations of $\delta g=\varepsilon \dot{g}$,

$$
\delta g=g\left(\varepsilon g^{-1} \dot{g}\right)=\left(\varepsilon \dot{g} g^{-1}\right) g .
$$

Thus applying Eq. (2.23) with $L=\varepsilon \dot{g} g^{-1}$, we find

$$
\delta \mathscr{L}=\operatorname{Tr}\left(\dot{x} x^{-1} \frac{d}{d t}\left(\varepsilon \dot{g} g^{-1}\right)\right) / 2 .
$$


Since

$$
\begin{aligned}
\dot{x} x^{-1} & =2 g A g^{-1} \text { and } \dot{g} g^{-1}=g\left(g^{-1} \dot{g}\right) g^{-1}, \\
\delta \mathscr{L} & =\dot{\varepsilon} \operatorname{Tr}(A(A+i B))+\varepsilon \operatorname{Tr}(A(\dot{A}+i \dot{B})) \\
& =\frac{d}{d t}\left(\varepsilon \operatorname{Tr} A^{2} / 2\right)+\dot{\varepsilon} \operatorname{Tr} A^{2} / 2,
\end{aligned}
$$

using (2.5) and (2.9). Comparing with Eq. (3.1) we see that $D$ vanishes. Thus condition $\alpha$ of Noether's Theorem is satisfied so that the generator of the time translations, namely the Hamiltonian, $H$, is conserved and given by the coefficient of $\dot{\varepsilon}$ as

$$
H=\operatorname{Tr} A^{2} / 2,
$$

when canonical variables are used. This is indeed positive by Eqs. (2.5) and (2.9) as mentioned earlier. Obviously it is conserved by Eqs. (2.7) and (2.11). So are the quantities

$$
H_{n}=\operatorname{Tr}\left(A^{n}\right) / n
$$

\section{The Fundamental Poisson Relation and the Yang-Baxter Equations}

So far we have worked with any simple non-compact Lie group $F$ equipped with a Cartan involution. Now we shall specialise $F$ to $G^{N}$, the normal real form of the complex simple Lie group whose Cartan matrix occurs in the Toda molecule equation (1.1). $g^{N}$ is the real Lie algebra generated by the usual Cartan subalgebra generators $H_{i}$ and the step operators $E_{\alpha}$.

$$
\begin{aligned}
{\left[H_{i}, H_{j}\right] } & =0, \quad i=1 \ldots r \\
{\left[H_{i}, E_{ \pm \alpha}\right] } & = \pm \alpha^{i} E_{ \pm \alpha}, \\
{\left[E_{\alpha}, E_{-\alpha}\right] } & =2 \alpha \cdot H / \alpha^{2}, \\
{\left[E_{\alpha}, E_{\beta}\right] } & =N_{\alpha \beta} E_{\alpha+\beta} \quad \alpha, \beta>0,
\end{aligned}
$$

together with other equations not needed explicitly. Notice that all structure constants are real, confirming that this is indeed a real Lie algebra. The Cartan involution is

$$
\sigma\left(H_{i}\right)=-H_{i}, \quad \sigma\left(E_{\alpha}\right)=-E_{-\alpha} .
$$

Then the various subspaces and subalgebras of $\mathbf{g}^{N}$ have the following bases:

$$
\left.\begin{array}{l}
\mathbf{k}=\left\{E_{\alpha}-E_{-\alpha}\right\}, \quad \alpha>0 \\
\mathbf{p}=\left\{E_{\alpha}+E_{-\alpha}, H_{i}\right\}, \quad \alpha>0, \quad i=1 \ldots r \\
\mathbf{a}=\left\{H_{i}\right\}, \quad i=1 \ldots r \\
\mathbf{n}=\left\{E_{\alpha}\right\}, \quad \alpha>0 .
\end{array}\right\}
$$

If we normalise our Killing form by $\operatorname{Tr}\left(H_{i} H_{j}\right)=\delta_{i j}$, it follows that

$$
\operatorname{Tr}\left(H_{i} E_{\alpha}\right)=0 \quad \text { and } \operatorname{Tr}\left(E_{\alpha} E_{-\beta}\right)=2 \delta_{\alpha \beta} / \alpha^{2} .
$$


It is easy now to check the Cartan property (2.4). We suspect that the work of this section can be generalized to any choice of $\sigma$, but we have not checked this completely.

In the last section we saw that the components of $x^{-1} \dot{x} / 2$ and $g^{-1} \dot{g}$ (in the $k=1$ gauge) constituted Noether charges with definite algebraic properties. Our first aim is to develop a new notation which expresses this clearly.

In the gauge $k=1, g$ is obtained by exponentiating the $H_{i}$ and $E_{\alpha}$ 's $(\alpha>0)$ (Eqs. 2.24, 4.3), and therefore we can expand

$$
g^{-1} \dot{g}=A+i B=\sum_{i} D_{i} H_{i}+\sum_{\alpha>0}\left(\alpha^{2} / 2\right) D_{\alpha} E_{\alpha}
$$

for some coefficients to be determined. As $A$ is the odd part of $g^{-1} \dot{g}$ under $\sigma$ (2.10) we have by (4.2),

$$
A=\sum_{i} D_{i} H_{i}+\sum_{\alpha>0}\left(\alpha^{2} / 2\right) D_{\alpha}\left(E_{\alpha}+E_{-\alpha}\right) / 2
$$

By (4.4)

$$
\begin{aligned}
& D_{i}=\operatorname{Tr}\left(A H_{i}\right)=-\operatorname{Tr}\left(A \sigma\left(H_{i}\right)\right), \\
& D_{\alpha}=2 \operatorname{Tr}\left(A E_{\alpha}\right)=-2 \operatorname{Tr}\left(A \sigma\left(E_{-\alpha}\right)\right) .
\end{aligned}
$$

Let us define

$$
D(T)=-\operatorname{Tr}(A \sigma(T)), \quad T \in \sigma(\mathbf{b}) .
$$

Then by the Noether charge algebra (3.6) and the fact that $\sigma$ is an automorphism of the Lie algebra

$$
\left\{D(T), \quad D\left(T^{\prime}\right)\right\}_{P B}=D\left(\left[T, T^{\prime}\right]\right), \quad T, T^{\prime} \in \sigma(\mathbf{b}) .
$$

Thus we have found

$$
g^{-1} \dot{g}=A+i B=D=\sum_{i} H_{i} D\left(H_{i}\right)+2 \sum_{\alpha>0}\left(\alpha^{2} / 2\right) E_{\alpha} D\left(E_{-\alpha}\right) .
$$

This combination will be called $D$, for short. Notice the factor 2 in the second term of (4.7).

Similarly we find that

$$
\begin{aligned}
\dot{x} x^{-1} / 2 & =-\sigma\left(x^{-1} \dot{x}\right) / 2 \\
& =\sum_{i} H_{i} X\left(H_{i}\right)+\sum_{\alpha>0}\left(\alpha^{2} / 2\right)\left(E_{\alpha} X\left(E_{-\alpha}\right)+E_{-\alpha} X\left(E_{\alpha}\right)\right)
\end{aligned}
$$

when the coefficients $X(L)$ satisfy Eqs. (3.9) and (3.10).

We see that the Hamiltonian $H$ (3.12) can be expressed in two alternative ways,

$$
\begin{aligned}
H & =\left[\sum_{i} D\left(H_{i}\right)^{2}+\sum_{\alpha>0} \alpha^{2} D\left(E_{-\alpha}\right)^{2}\right] / 2 \\
& =\left[\sum_{i} X\left(H_{i}\right)^{2}+\sum_{\alpha>0}\left(\alpha^{2} / 2\right) X\left(E_{\alpha}\right) X\left(E_{-\alpha}\right)\right] / 2 .
\end{aligned}
$$

Following a notation introduced in statistical physics $[16,1]$, Eq. (4.6) can be 
written in another way,

$$
\{D \otimes D\}_{P B}=-[\mathbb{P}, D \otimes 1+1 \otimes D] .
$$

Faddeev calls a relation like this a "fundamental Poisson relation" [1]. Apart from a factor - 2 (a 2 to compensate for a different definition of Lagrangian and a minus for convenience), $\mathbb{P}$ is the operator constructed by Olive and Turok [14].

$$
\mathbb{P}=\sum_{\alpha>0}\left(\alpha^{2} / 2\right)\left(E_{\alpha} \otimes E_{-\alpha}-E_{-\alpha} \otimes E_{\alpha}\right)=\mathbb{C}_{+}-\mathbb{C}_{-} .
$$

Equation (4.10) is proven in Appendix II following the methods of Olive and Turok [14] and using properties of root systems of Lie algebras. In that work it is also useful to define an operator

$$
\begin{aligned}
\mathbb{C} & =\sum_{i} H_{i} \otimes H_{i}+\sum_{\alpha>0}\left(\alpha^{2} / 2\right)\left(E_{\alpha} \otimes E_{-\alpha}+E_{-\alpha} \otimes E_{\alpha}\right) \\
& =\mathbb{C}_{0}+\mathbb{C}_{+}+\mathbb{C}_{-} .
\end{aligned}
$$

It is possible to rewrite $(4.10)$ as

$$
\{D \otimes D\}_{P B}=-[\mathbb{P}+\mathbb{C}, D \otimes 1+1 \otimes D],
$$

as the $\mathbb{C}$ contribution vanishes identically. We now show that this equation has the same structure as the Yang-Baxter equation. This is because

$$
\mathbb{P}+\mathbb{C}=\mathbb{C}_{0}+2 \mathbb{C}_{+}=\sum_{i} H_{i} \otimes H_{i}+2 \sum_{\alpha>0}\left(\alpha^{2} / 2\right) E_{\alpha} \otimes E_{-\alpha},
$$

and the structure of this expression is very similar to that of $D$ in Eq. (4.7). We can develop this resemblance by introducing a triple notation with three spaces. The first and third spaces are occupied by the left and right Lie algebra generators in (4.10) and (4.13). The middle entry is the space of dynamical variables in which the bracket operation is the Poisson bracket. With the suffices referring to these three spaces we define

$$
\begin{aligned}
& D_{12}=D \otimes 1=2 \sum_{\alpha>0}\left(\alpha^{2} / 2\right) E_{\alpha} \otimes D\left(E_{-\alpha}\right) \otimes 1+\sum_{i} H_{i} \otimes D\left(H_{i}\right) \otimes 1, \\
& D_{32}=1 \otimes D=2 \sum_{\alpha>0}\left(\alpha^{2} / 2\right) 1 \otimes D\left(E_{-\alpha}\right) \otimes E_{\alpha}+\sum_{i} 1 \otimes D\left(H_{i}\right) \otimes H_{i}, \\
& D_{13}=\mathbb{P}+\mathbb{C}=2 \sum_{\alpha>0}\left(\alpha^{2} / 2\right) E_{\alpha} \otimes 1 \otimes E_{-\alpha}+\sum_{i} H_{i} \otimes 1 \otimes H_{i} .
\end{aligned}
$$

Then Eq. (4.13) can be written

$$
\left\{D_{12}, D_{32}\right\}_{P B}=-\left[D_{13}, D_{12}+D_{32}\right]
$$

or

$$
\left\{D_{12}, D_{32}\right\}_{P B}+\left[D_{13}, D_{12}\right]+\left[D_{13}, D_{32}\right]=0 \text {. }
$$

The quantum version of this is the Yang-Baxter equation $[15,16]$, which is the infinitesimal version of the triangle equation. We have learnt how to obtain this from 
something rather geometrical, namely the geodesic motion of a particle on a noncompact Reimannian symmetric space.

Finally we see how to extract from (4.10) the Poisson brackets of the individual components of $A$ and $B$ by using the involution $\sigma$. Let us define $\sigma_{L}$ and $\sigma_{R}$ as $\sigma$ acting respectively on the left and right entries of $\{D \otimes D\}$. By considering $\left(1-\sigma_{L}-\right.$ $\sigma_{R}+\sigma_{L} \sigma_{R}$ ) acting on Eq. (4.10) we find

$$
\begin{aligned}
\{A \otimes A\}_{P B} & =-\left[\frac{\mathbb{P}+\mathbb{R}}{2}, A \otimes 1\right]-\left[\frac{\mathbb{P}-\mathbb{R}}{2}, 1 \otimes A\right] \\
& =-(1 / 2)[\mathbb{P}, A \otimes 1+1 \otimes A]-(1 / 2)[\mathbb{R}, A \otimes 1-1 \otimes A],
\end{aligned}
$$

where

$$
\mathbb{R}=\sigma_{L} \mathbb{P}=-\sigma_{R} \mathbb{P}=\sum_{\alpha>0}\left(\alpha^{2} / 2\right)\left(E_{\alpha} \otimes E_{\alpha}-E_{-\alpha} \otimes E_{-\alpha}\right) .
$$

Similarly we find

$$
\begin{aligned}
& \{A \otimes B\}_{P B}=-\left[\frac{\mathbb{P}-\mathbb{R}}{2}, 1 \otimes B+B \otimes 1\right] \\
& \{B \otimes, B\}_{P B}=-\left[\frac{\mathbb{P}-\mathbb{R}}{2}, A \otimes 1\right]-\left[\frac{\mathbb{P}+\mathbb{R}}{2}, 1 \otimes A\right] .
\end{aligned}
$$

Using (4.16) and the methods of ref. [14] it is possible to prove that

$$
\left\{A, \operatorname{Tr} A^{N} / N\right\}_{P B}=-i\left[B_{N}, A\right],
$$

where

$$
i B_{N}=\operatorname{Tr}_{R}\left(A^{N-1} \frac{(\mathbb{P}+\mathbb{R})}{2}\right)=\operatorname{Tr}_{R}\left(A^{N-1} \mathbb{P}\right),
$$

with the last equality holding only if $\operatorname{Tr}\left(A^{N}\right)$ is non-zero. From (4.19) it follows that the quantities $\operatorname{Tr} A^{N}$ are in involution, i.e.:

$$
\left\{\operatorname{Tr} A^{N}, \operatorname{Tr} A^{M}\right\}_{P B}=0 .
$$

These results mean that any of the quantities $\operatorname{Tr} A^{N} / N$ can be used as a new Hamiltonian and that $A, B_{N}$ will constitute the corresponding Lax pair. Of course $B_{2}=B$.

\section{Reduction to the Toda Molecule System}

The final step is to find the precise constraints on the geodesic trajectories necessary to produce the Toda molecule equation (1.1) with the correspondingly constrained quantities $A$ and $B$ satisfying the usual Lax pair and fundamental Poisson relations of that system. The constraints have to be self consistent and one possibility is to constrain

$$
X\left(E_{\alpha}\right)=D\left(E_{-\alpha}\right)=0, \quad \alpha \text { any positive non-simple root. }
$$


These equations are self consistent since the quantities put to zero in (5.1) form a closed subalgebra under the Poisson brackets by Eqs. (3.10), (3.11), (4.5) and (4.6). The quantities $X\left(E_{\alpha}\right)$ are conserved and so by putting them equal to zero when $\alpha$ is a positive but non-simple root we are choosing initial conditions on the trajectory. It remains to see how these conditions imply the vanishing of the $D\left(E_{-\alpha}\right)$ in (5.1) and how this leads to the Toda equations (1.1). To do this we introduce explicit (horospheric) coordinates $\left(\phi_{a}, \rho_{\alpha}\right)$ for the symmetric space,

$$
\begin{aligned}
g & =n a, \quad a=\exp \left(\sum_{a} \phi_{a} H_{a} / 2\right), \quad n=\exp \left(\sum_{\alpha>0} \rho_{\alpha} E_{\alpha}\right), \\
n^{-1} \dot{n} & =\sum_{\alpha>0} E_{\alpha} V_{\alpha}, \quad V_{\alpha}=\sum_{\beta>0} \dot{\rho}_{\beta} M_{\beta \alpha}(\rho) .
\end{aligned}
$$

It is understood that the greek indices refer to positive roots, whereas the Latin indices refer to the $r$ simple roots. Thus

$$
H_{a}=2 a \cdot H / a^{2} \text {, where } a \text { is a simple root. }
$$

The variables $\phi_{a}$ will be the same as those appearing in (1.1). Up to now we have managed to keep our notation relatively simple by not introducing specific coordinates. More concrete proofs of our previous Poisson bracket relations can be obtained using these coordinates (5.2), but we leave this as an exercise for the reader.

In terms of the new variables (5.2),

$$
g^{-1} \dot{g}=A+i B=a^{-1} \dot{a}+a^{-1} n^{-1} \dot{n} a=(1 / 2) \sum_{a} \dot{\phi}_{a} H_{a}+\sum_{\alpha>0} \exp \left(-K_{\alpha b} \phi_{b} / 2\right) V_{\alpha} E_{\alpha},
$$

using the commutators (4.1) and introducing

$$
K_{\alpha b}=2 \alpha \cdot b / b^{2} .
$$

When $\alpha$ as well as $b$ is a simple root, this forms the Cartan matrix occurring in (1.1). Also we see

$$
x^{-1} \dot{x}=\left(n^{+}\right)^{-1}\left[a^{-2} n^{-1} \dot{n} a^{2}+2 a^{-1} \dot{a}+\dot{n}^{+}\left(n^{+}\right)^{-1}\right] n^{+},
$$

where for any element $g$ of $G^{N}$ we define $g^{+}=\sigma(g)^{-1}$.

It is difficult to evaluate this further, but it is easy to see that if $\gamma$ is one of the highest positive roots whose step operator $E_{\gamma}$ occurs in $n^{-1} \dot{n}$ in the sense that $E_{\gamma+\alpha}$ does not occur for any positive root $\alpha$, then the coefficient of $E_{\gamma}$ in $x^{-1} \dot{x}$ is simply $V_{\gamma} \exp \left(-K_{\gamma b} \phi_{b}\right)$ (as it is the same as the coefficient of $E_{\gamma}$ in $a^{-2} n^{-1} \dot{n} a^{2}$, since the $n^{+}$'s do not affect this term). As $x^{-1} \dot{x}$ is conserved, so is this coefficient. Hence, if $V_{\gamma}$ vanishes initially, it does so for all time. So therefore do the coefficients of $E_{\gamma}$ in $n^{-1} \dot{n}$ and $g^{-1} \dot{g}$ since they are, respectively by (4.7), (5.2) and (5.3), $V_{\gamma}$ and $V_{\gamma} \exp \times$ $\left(-K_{\gamma b} \phi_{b} / 2\right)$. In particular $D\left(E_{-\gamma}\right)$ vanishes. This argument can now be repeated for any of the remaining highest roots until only step operators for simple roots $a$ remain in $n^{-1} \dot{n}$ and $g^{-1} \dot{g}$. The coefficient of $E_{a}$ in $x^{-1} \dot{x}$ is then $V_{a} \exp \left(-K_{a b} \phi_{b}\right)$ and constant. By Eq. (4.8) we have

$$
a^{2} X\left(E_{a}\right)=V_{a} \exp \left(-K_{a b} \phi_{b}\right), \quad \text { a simple, }
$$


But by Eqs. (5.3) and (4.7) the coefficient of $E_{a}$ in $g^{-1} \dot{g}$ is

$$
a^{2} D\left(E_{-a}\right)=V_{a} \exp \left(-K_{a b} \phi_{b} / 2\right)=a^{2} X\left(E_{a}\right) \exp \left(K_{a b} \phi_{b} / 2\right)
$$

by the preceding result. In this way we have integrated the $\rho$ equations of motion by choosing special initial conditions leaving as the only degrees of freedom the $\phi$ variables. By (5.5) we now have

$$
\bar{A}+i \bar{B}=(1 / 2) \sum_{a} \dot{\phi}_{a} H_{a}+\sum_{a} E_{a} a^{2} X\left(E_{a}\right) \exp \left(K_{a b} \phi_{b} / 2\right)
$$

where now both sums extend over simple roots only. The resultant $\bar{A}$ and $\bar{B}$ form the Lax pair for the Toda molecule equation,

$$
\frac{d^{2}}{d t^{2}} \phi_{a}=-a^{4} X\left(E_{a}\right)^{2} \exp \left(K_{a b} \phi_{b}\right)
$$

Thus the constants $a^{2} X\left(E_{a}\right)$ constitute coupling constants. To obtain precisely (1.1) we assign these constants the value unity. Then (5.6) yields the usual Lax pair for Eqs. (1.1) (apart from a minus sign in $B$ when compared to ref. [14] owing to a different sign in (2.14)). These substitutions for the integrals of motion $X\left(E_{\alpha}\right)$ can be made directly into the equations of motion or the Hamiltonians (but not the Lagrangian). All the Poisson bracket relations remain valid with these substitutions.

We now show how the fundamental Poisson relations (4.10), (4.16), and (4.18) reduce to the ones obtained by Olive and Turok [14].

In Appendix II it is shown that for any positive root $\beta$

$$
\begin{aligned}
& {\left[\mathbb{P}, \frac{\left(E_{\beta}+E_{-\beta}\right)}{2} \otimes 1+1 \otimes \frac{\left(E_{\beta}+E_{-\beta}\right)}{2}\right]} \\
& =\left(\beta^{2} / 2\right)\left(H_{\beta} \otimes \frac{\left(E_{\beta}+E_{-\beta}\right)}{2}-\frac{\left(E_{\beta}+E_{-\beta}\right)}{2} \otimes H_{\beta}\right) \\
& \quad+(1 / 2) \sum_{\substack{\alpha, \gamma>0 \\
\alpha+\gamma=\beta}}\left(\alpha^{2} \gamma^{2} / \beta^{2}\right) N_{\alpha \gamma}\left(E_{\alpha} \otimes E_{\gamma}+E_{-\alpha} \otimes E_{-\gamma}\right) .
\end{aligned}
$$

When $A$ is reduced by (5.5) only step operators for simple roots occur. When $\beta$ is simple it cannot be expressed as a sum of two positive roots $\alpha$ and $\gamma$, and hence the last term in the equation vanishes. The remaining term is odd under either $\sigma_{L}$ or $\sigma_{R}$ by (4.2). Hence so is the left-hand side. Thus by (4.17),

$$
\left[\mathbb{P}, \frac{\left(E_{\beta}+E_{-\beta}\right)}{2} \otimes 1+1 \otimes \frac{\left(E_{\beta}+E_{-\beta}\right)}{2}\right]=\left[\mathbb{R}, \frac{\left(E_{\beta}+E_{-\beta}\right)}{2} \otimes 1-1 \otimes \frac{\left(E_{\beta}+E_{-\beta}\right)}{2}\right] .
$$

This leads to the fact that after the reduction the two terms on the right-hand side of (4.16) become equal, yielding

$$
\{\bar{A} \otimes, \bar{A}\}=-[\mathbb{P}, \bar{A} \otimes 1+1 \otimes \bar{A}]
$$

which is a result of Olive and Turok. Similarly $\{\bar{B} \otimes \bar{B}\}=0$. 


\section{Conclusions}

We have succeeded in finding some sort of geometric principle underlying the Poisson bracket relations so essential to integrable theories, at least in the class of prototypes theory given by the Toda molecule equations (1.1).

The point has been that the non-compact Riemannian symmetric spaces considered have a hidden group theoretical structure owing to the Iwasawa decomposition $g=n a k$, which enables us to parametrise the points of the symmetric space by $n a$, which are elements of the group $\mathbf{B}=\mathbf{N A}$. The Lie algebra of $\mathbf{B}$ is realized by the Poisson brackets of the components of $A$, where $A$ and $B$ satisfy the Lax pair equation describing geodesic motion on the symmetric space. This algebra leads to the "fundamental Poisson bracket" or "Yang-Baxter" equations which are of importance in a large class of integrable systems and statistical physical models $[1,16]$. We showed in detail how a reduction procedure involving a subclass of geodesic trajectories lead to the Toda molecule equations and their integrability properties. There is a Toda molecule equation associated with each simple Lie algebra and our analysis applied uniformly to all these equations.

There are many other integrable dynamical systems not covered here and we wonder if our analysis can be generalized to them. Indeed Olshanetsky and Perelomov have conjectured that all integrable equations correspond to geodesic motions on a suitable space, presumably a symmetric space or a generalization $[8,10]$.

For example there are other non-compact Riemannian symmetric spaces besides those leading to the Toda molecule equations and much of our analysis (if not all) applies to them, too. We do not know what equations the analogous reduction procedure could lead to.

There are other algebras beside Lie algebras, for example Kac-Moody algebras. There are Toda lattice equations associated with these with very similar integrability properties. The $\mathbb{P}$ operator entering the fundamental Poisson bracket relations has a very similar structure to the one considered here in terms of the root system of the algebra [14]. The infinite nature of this root system accounts for the appearance of a "spectral parameter," which also occurs in many other systems. It is possible that this could likewise be understood in terms of the Iwasawa decomposition for the Kac-Moody group. Since these groups are infinite dimensional, the necessary mathematical theory does not exist yet as far as we know.

It is highly desirable to apply the ideas of this paper to such systems, to the two dimensional field theory versions of these equations which are also believed to be integrable, and also the quantum versions of these theories.

It is quite possible that this ideas will link up with the work of the Kyoto group [20] who has developed an algebraic theory of soliton equations starting from a somewhat different point of view.

\section{Appendix I: Noether's Theorem}

This is a standard theorem, yet we have not succeeded in finding in the literature a statement and proof sufficiently explicit for the use made in the text. Here we present 
the version we use, followed by an outline proof. We consider a nonsingular dynamical system with a finite number of coordinates $q_{1} \ldots q_{N}$.

Theorem A. Consider the variation,

$$
\delta q_{i}=\varepsilon(t) F_{i}(q, \dot{q}, t), \quad i=i \ldots N,
$$

(with $\delta \dot{q}_{i}=d / d t\left(\delta q_{i}\right)$ as is usual in Lagrangian theory) and suppose that without using any equations of motion the corresponding response of the Lagrangian is found to be

$$
\delta \mathscr{L}=\frac{d}{d t}(\varepsilon X)+\dot{\varepsilon} Q+\varepsilon D,
$$

where $X, Q$ and $D$ may depend on $q, \dot{q}$ and $t$ (but not accelerations). Then

(i) $d Q / d t=D$ by virtue of the equation of motion,

(ii) $Q=\sum_{i=1}^{N} p_{i} F_{i}-X$,

(iii) $Q$ can be expressed in terms of canonical variables $q_{i}$ and $p_{i}$ (with velocities eliminated) and

$$
\left\{q_{i}, \varepsilon Q\right\}_{P B}=\delta q_{i}, \quad\left\{p_{i}, \varepsilon Q\right\}_{P B}=\delta p_{i}-\varepsilon \frac{\partial D}{\partial \dot{q}_{i}},
$$

where $\delta p_{i}$ is calculated directly from the variations of the coordinates and velocities using the equations of motion if necessary, and $\varepsilon$ is taken be constant.

Corollary. There is an arbitrariness in the definition of $Q, X$ and $D$. If we replace

$$
X \rightarrow X-G, \quad Q \rightarrow Q+G, \quad D \rightarrow D+\dot{G},
$$

where $G=G(q, t)$, the original definition and all the subsequent properties still hold good. If we can choose $G$ so that $D$ becomes velocity independent, then the variation (with $\dot{\varepsilon}=0$ ) is seen to be canonical with $Q$ the infinitesimal generator. It still has an ambiguity which is an additive function of time. If $D$ can be chosen to be zero, $Q$ is conserved and certainly generates the infinitesimal transformation. Its ambiguity is just an additive constant.

Theorem B for Velocity Independent Variations. If the variations are velocity independent, then so is $X$, which can therefore be put to zero by a suitable choice of G. Then

$$
Q=\sum_{i} p_{i} F_{i}
$$

If there are three variations of this type, $\delta, \delta^{\prime}$ and $\delta^{\prime \prime}$ satisfying, $\left[\delta, \delta^{\prime}\right] q_{i}=\delta^{\prime \prime} q_{i}$, then $\left\{Q, Q^{\prime}\right\}_{P B}=-Q^{\prime \prime}$.

Outline Proof of Theorem A. (1) follows immediately from Hamilton's action principle. Alternatively if we evaluate $\delta \mathscr{L}$ directly by calculation we get 


$$
\delta \mathscr{L}=\frac{\partial \mathscr{L}}{\partial q_{i}} \delta q_{i}+\frac{\partial \mathscr{L}}{\partial \dot{q}_{i}} \delta \dot{q}_{i}=\frac{d}{d t}\left(\varepsilon p_{i} F_{i}\right)+\varepsilon\left(\frac{\partial \mathscr{L}}{\partial q_{i}}-\dot{p}_{i}\right) F_{i}
$$

Comparing with (I.1), result (ii) follows by equating coefficients of $\dot{\varepsilon}$, and (i) by equating coefficients of $\varepsilon$.

We now obtain an important relation by comparing the occurrence of the acceleration $\ddot{q}_{i}$ in the two expressions for $\delta \mathscr{L}$. It occurs only once, linearly, in the terms $\varepsilon p_{j} \partial F_{j} / \partial \dot{q}_{i} \ddot{q}_{i}$ and $\varepsilon \partial X / \partial \dot{q}_{i} \ddot{q}_{i}$ respectively. Since no equations of motion are used, these terms are equal for all accelerations. Hence

$$
\frac{\partial X}{\partial \dot{q}_{i}}=\sum_{j} p_{j} \frac{\partial F_{j}}{\partial \dot{q}_{i}}
$$

Now we examine the structure of $Q$. By (ii),

$$
\begin{aligned}
d Q= & \sum_{i} d p_{i} F_{i}+\sum_{i, j}\left(p_{j} \frac{\partial F_{j}}{\partial q_{i}}-\frac{\partial X}{\partial q_{i}}\right) d q_{i}+\sum_{i, j}\left(p_{j} \frac{\partial F_{j}}{\partial \dot{q}_{i}}-\frac{\partial X}{\partial \dot{q}_{i}}\right) d \dot{q}_{i} \\
& +\sum_{i}\left(p_{i} \frac{\partial F_{i}}{\partial t}-\frac{\partial X}{\partial t}\right) d t
\end{aligned}
$$

and by (I.4) we see that the coefficient of $d \dot{q}$ vanishes, thereby confirming the first assertion in (iii). Further $\partial Q / \partial p_{i}=F_{i}$, yielding the second part of (iii), while

$$
\frac{\partial Q}{\partial q_{i}}=\sum_{j} p_{j} \frac{\partial F_{j}}{\partial q_{i}}-\frac{\partial X}{\partial q_{i}}=-\left\{p_{i}, Q\right\}_{P B}
$$

Finally we calculate $\delta p_{i}$, where $p_{i}=\partial \mathscr{L}(q, \dot{q}, t) / \partial \dot{q}_{i}$. Using (I.1) and taking $\varepsilon$ constant, we get

$$
\delta p_{i}=\varepsilon \frac{\partial}{\partial \dot{q}_{i}}(\dot{X}+D)-\varepsilon \frac{\partial \mathscr{L}}{\partial q_{i}} \frac{\partial F_{j}}{\partial \dot{q}_{i}}-\varepsilon \frac{\partial \mathscr{L}}{\partial \dot{q}_{i}} \frac{\partial \dot{F}_{j}}{\partial \dot{q}_{i}} .
$$

Using (I.4), (I.5) and the fact that for any function $f(q, \dot{q}, t)$

$$
\frac{\partial \dot{f}}{\partial \dot{q}_{i}}=\frac{d}{d t}\left(\frac{\partial f}{\partial \dot{q}_{i}}\right)+\frac{\partial f}{\partial q_{i}}+\frac{\partial f}{\partial \dot{q}_{i}} \frac{\partial \ddot{q}_{j}}{\partial \dot{q}_{i}},
$$

we obtain

$$
\delta p_{i}=\varepsilon\left\{p_{i}, Q\right\}_{P B}+\varepsilon \frac{\partial D}{\partial \dot{q}_{i}}+\varepsilon \sum_{j} \frac{\partial F_{j}}{\partial \dot{q}_{i}}\left(\dot{p}_{j}-\frac{\partial \mathscr{L}}{\partial q_{j}}\right) .
$$

This yields the last part of (iii) on using Lagrange's equations of motion. If the variation of the $j^{\text {th }}$ coordinate is velocity independent, it is unnecessary to use the corresponding equation of motion.

Proof of Corollary and Theorem B. The corollary is self evident once it is recognized $G$ must be velocity independent in order to keep $D$ acceleration independent.

If the variations are velocity independent as in Theorem $B$, it follows from (1.4) that $X$ is too. Then we can choose the $G$ of the corollary equal to $X$ in order to make 
the subsequent $X$ vanish so that by (ii) $Q$ is as stated. Of course $D$ may not then be velocity independent, but this is not relevant to the final statement. If $\delta, \delta^{\prime}, \delta^{\prime \prime}$ are three velocity independent variations related as stated, we find

$$
F_{i}^{\prime \prime}=\sum_{j}\left(\frac{\partial F_{i}^{\prime}}{\partial q_{j}} F_{j}-\frac{\partial F_{i}}{\partial q_{j}} F_{j}^{\prime}\right), \quad \varepsilon^{\prime \prime}=\varepsilon \varepsilon^{\prime} .
$$

Then the stated Poisson bracket follows by direct calculation using this relation.

\section{Appendix II}

In this appendix we prove relation (4.10) following the methods of ref. [14].

Using the commutation relations (4.1) it is easy to check that the operator $\mathbb{P}$ defined by (4.11) satisfies:

$$
\left[\mathbb{P}, 1 \otimes H_{a}+H_{a} \otimes 1\right]=0 .
$$

In order to evaluate the commutator of $\mathbb{P}$ with step operators we make use of the Casimir like operator $\mathbb{C}$ defined by (4.12). It has the property of commuting with any generator $T$ of the algebra, [14],

$$
[\mathbb{C}, 1 \otimes T+T \otimes 1]=0 .
$$

Using (4.1) we have, for any positive $\operatorname{root} \beta$, that $\left(r=\operatorname{rank}\right.$ of $\left.G^{N}\right)$ :

$$
\begin{aligned}
{\left[\mathbb{C}_{0}, 1 \otimes E_{\beta}+E_{\beta} \otimes 1\right] } & =\sum_{i=1}^{r}\left(H_{i} \otimes \beta_{i} E_{\beta}+\beta_{i} E_{\beta} \otimes H_{i}\right) \\
& =\left(\beta^{2} / 2\right)\left(H_{\beta} \otimes E_{\beta}+E_{\beta} \otimes H_{\beta}\right),
\end{aligned}
$$

Where $\mathbb{C}_{0}=\sum_{i} H_{i} \otimes H_{i}$ and $H_{\beta}=2 \beta \cdot H / \beta^{2}$.

Since $\mathbb{C}=\mathbb{C}_{0}+\mathbb{C}_{+}+\mathbb{C}_{-}$, we get from (II.2) and (II.3) that:

$$
\left[\mathbb{C}_{+}+\mathbb{C}_{-}, 1 \otimes E_{\beta}+E_{\beta} \otimes 1\right]=-\left(\beta^{2} / 2\right)\left(H_{\beta} \otimes E_{\beta}+E_{\beta} \otimes H_{\beta} .\right)
$$

But we also have

$$
\left[\mathbb{C}_{+}, 1 \otimes E_{\beta}+E_{\beta} \otimes 1\right]=\sum_{\alpha>0}\left(\alpha^{2} / 2\right)\left(E_{\alpha} \otimes\left[E_{-\alpha}, E_{\beta}\right]+\left[E_{\alpha}, E_{\beta}\right] \otimes E_{-\alpha}\right),
$$

and

$$
\left[\mathbb{C}_{-}, 1 \otimes E_{\beta}+E_{\beta} \otimes 1\right]=\sum_{\alpha>0}\left(\alpha^{2} / 2\right)\left(E_{-\alpha} \otimes\left[E_{\alpha}, E_{\beta}\right]+\left[E_{-\alpha}, E_{\beta}\right] \otimes E_{\alpha}\right) .
$$

Since (II.4) contains terms proportional to the product of Cartan subalgebra generators with positive step operators only, we conclude that the sum of terms proportional to $E_{\gamma} \otimes E_{-\gamma^{\prime}}\left(\gamma, \gamma^{\prime}>0\right)$ in (II.5) must vanish since we do not have such a contribution in (II.6). Similarly the sum of terms proportional to $E_{-\gamma} \otimes E_{\gamma^{\prime}}\left(\gamma, \gamma^{\prime}>0\right)$ in (II.6) must also vanish since we do not have such a contribution in (II.5). In addition we conclude that the sum of terms proportional to the product of positive 
step operators in (II.5) and (II.6) must vanish, i.e.,

$$
\sum_{0<\alpha<\beta}\left(\alpha^{2} / 2\right)\left(E_{\alpha} \otimes\left[E_{-\alpha}, E_{\beta}\right]+\left[E_{-\alpha}, E_{\beta}\right] \otimes E_{\alpha}\right)=0 .
$$

Therefore we get that

$$
\begin{aligned}
{\left[\mathbb{C}_{+}-\mathbb{C}_{-}, 1 \otimes E_{\beta}+E_{\beta} \otimes 1\right]=} & \left(\beta^{2} / 2\right)\left(H_{\beta} \otimes E_{\beta}-E_{\beta} \otimes H_{\beta}\right) \\
& +2 \sum_{0<\alpha<\beta}\left(\alpha^{2} / 2\right) E_{\alpha} \otimes\left[E_{-\alpha}, E_{\beta}\right] .
\end{aligned}
$$

Now, suppose $\alpha, \beta$ and $\gamma$ are positive roots and $\beta=\gamma+\alpha$, then: $\left[E_{-\alpha}, E_{\beta}\right]=a E_{\gamma}$, and

$$
\operatorname{Tr}\left(\left[E_{-\alpha}, E_{\beta}\right] E_{-\gamma}\right)=2 a / \gamma^{2}=\operatorname{Tr}\left(\left[E_{-\gamma}, E_{-\alpha}\right] E_{\gamma}\right)=\left(2 / \beta^{2}\right) N_{\alpha \gamma},
$$

and so

$$
a=\left(\gamma^{2} / \beta^{2}\right) N_{\alpha \gamma}
$$

where we have used (4.4), and the commutator $\left[E_{-\gamma}, E_{-\alpha}\right]$ was evaluated by applying the automorphism (4.2) to the last equation in (4.1).

Since $\mathbb{P}=\mathbb{C}_{+}-\mathbb{C}_{-}$, we have:

$$
\begin{aligned}
{\left[\mathbb{P}, 1 \otimes E_{\beta}+E_{\beta} \otimes 1\right]=} & \left(\beta^{2} / 2\right)\left(H_{\beta} \otimes E_{\beta}-E_{\beta} \otimes H_{\beta}\right) \\
& +\sum_{\substack{\alpha, \gamma>0 \\
\beta=\alpha+\gamma}}\left(\alpha^{2} \gamma^{2} / \beta^{2}\right) N_{\alpha \gamma} E_{\alpha} \otimes E_{\gamma} .
\end{aligned}
$$

From (4.7), (II.1) and (II.10) we get

$$
\begin{aligned}
{[\mathbb{P}, 1 \otimes D+D \otimes 1]=} & 2 \sum_{\beta>0}\left(\beta^{2} / 2\right)^{2} D\left(E_{-\beta}\right)\left(H_{\beta} \otimes E_{\beta}-E_{\beta} \otimes H_{\beta}\right) \\
& -\sum_{\beta>0} \sum_{\substack{\alpha, \gamma>0 \\
\beta=\alpha+\gamma}} \alpha^{2} \gamma^{2} D\left(\left[E_{-\alpha}, E_{-\gamma}\right]\right) E_{\alpha} \otimes E_{\gamma} .
\end{aligned}
$$

Using (4.6) and (4.7) we conclude (4.10) is true.

The commutator between $\mathbb{P}$ and step operators corresponding to negative roots can be easily obtained from (II.10) by making use of the involutive automorphism $\sigma$. From (4.2) and the fact that $\sigma_{R} \sigma_{L} \mathbb{P}=-\mathbb{P}$ we get from (II.10) that

$$
\begin{aligned}
{\left[\mathbb{P}, 1 \otimes E_{-\beta}+E_{-\beta} \otimes 1\right]=} & \left(\beta^{2} / 2\right)\left(H_{\beta} \otimes E_{-\beta}-E_{-\beta} \otimes H_{\beta}\right) \\
& +\sum_{\substack{\alpha, \gamma>0 \\
\beta=\alpha+\gamma}}\left(\alpha^{2} \gamma^{2} / \beta^{2}\right) N_{\alpha \gamma} E_{-\alpha} \otimes E_{-\gamma} .
\end{aligned}
$$

Acknowledgements. We wish to thank John Gipson for showing us his thesis. We are both grateful for hospitality during the early part of this work to the Mathematics and Physics Department of the University of Virginia. D. I. Olive is also grateful to the Institute for Advanced Study there.

\section{References}

1. Faddeev, L.: Integrable models in $1+1$ dimensional quantum field theory In: The Proceedings of the Ecole d'Ete de Physique Theorique, Les Houches 1982 Amsterdam: North Holland 
2. Kulish, P. P., Sklyanin, E. K.: Quantum spectral transform method, recent developments, p. 61. (In: Integrable quantum field theories. eds. Hietarinta, J., Montonen C. (eds) Lecture Notes in Physics, Vol. 151, Berlin, Heidelberg, New York: Springer 1982

3. Goddard, P., Nuyts, J., Olive, D.: Gauge theories and magnetic charges, Nucl. Phys. B125, 1-28 (1977). Montonen, C., Olive, D.: Magnetic monopoles as gauge particles? Phys. Lett. 72B, 117-120 (1977)

4. Olive, D.: Magnetic monopoles and electromagnetic duality conjectures. In: Monopoles in quantum field theory. eds. Craigie, N. S., Goddard, P., Nahm W. (eds) (Singapore, World Scientific 1982)

5. Leznov, A. M., Saveliev, M. V.: Spherical symmetric equations in gauge theories for an arbitrary semisimple compact Lie group Phys. Lett. 79B, 294-296 (1978); Representation theory and integration of non-linear spherically symmetric equations to gauge theories, Commun. Math. Phys. 74, 111-118 (1980)

6. Ganoulis, N., Goddard, P., Olive D.: Self-dual monopoles and the Toda molecules, Nucl. Phys. B205, [FS5], 601-636 (1982)

7. Leznov, A. M., Saveliev, M. V.: Representation of zero curvature for the system of non-linear partial differential equations $x_{\alpha, z z}=\exp (k x)_{\alpha}$ and its integrability, Lett. Math. Phys. 3, $489-494$ (1979)

8. Olshanetsky, M. A., Perelomov, A. M.: Explicit solutions of classical generalized Toda models. Inventiones Math. 54, 261-269 (1979); The Toda chain as a reduced system, Theor. Math. Phys. 45, $843-854$ (1980)

9. Kostant, B.: The solution to a generalized Toda lattice and representation theory, Adv. Math. 34, 195-338 (1979)

10. Olshanetsky, M. A., Perelomov, A. M.: Classical integrable finite dimensional systems related to Lie algebras, Phys. Rep. 71, 313-400 (1981)

11. Helgason, S.: Differential geometry, Lie groups and symmetric spaces. New York: Academic Press 1978

12. D'Auria, R., Regge, T., Sciuto, S.: Group theoretical construction of two-dimensional models with inifinite sets of conservation laws, Nucl. Phys. B171, 167-188 (1980); A general scheme for bidimensional models with associated linear set. Phys. Lett. 89B, 363-366 (1980)

13. Cremmer, E., Julia, B.: The SO(8) supergravity. Nucl. Phys. B159, 141-212 (1979)

14. Olive, D., Turok N.: Algebraic structure of Toda systems. Nucl. Phys. B220 [FS8], 491-507 (1983)

15. Yang, C. N.: Some exact results for the many-body problem in one dimension with repulsive deltafunction interaction. Phys. Rev. Lett. 19, 1312-1315 (1967)

16. Baxter, R. J.: Partition function of the eight-vertex lattice model. Ann. Phys. 70, 193-228 (1972); Exactly solved models in statistical mechanics. (New York: Academic Press 1982)

17. Eichenherr, H., Forger, M.: More about non-linear sigma models on symmetric spaces. Nucl. Phys. B164, 528-535 (1980)

18. Gipson, J. M.: The quantum projection method and completely integrable systems, Thesis, Yale University (1982)

19. Lax, P.: Integrals of non-linear equations of evolution and solitary waves, Comm. Pure Appl. Math. 21, 467-490 (1968)

20. Jimbo, M., Miwa, T.: Solitons and infinite dimensional algebras, Publ. RIMS, Kyoto University, 19, 943-1001 (1983)

Communicated by A. Jaffe

Received August 2, 1984; in revised form November 26, 1984 